\title{
Psicologia em Ação no SUS: a Interdisciplinaridade Posta à Prova
}

Psychology In Action At SUS: Interdisciplinarity Tested

Psicología En Acción En El SUS: La Interdisciplinaridad Puesta A Prueba

Leandra Lúcia Moraes Couto, Polyana Barbosa

Schimith \& Maristela Dalbello-Araujo

Universidade Federal do Espírito Santo
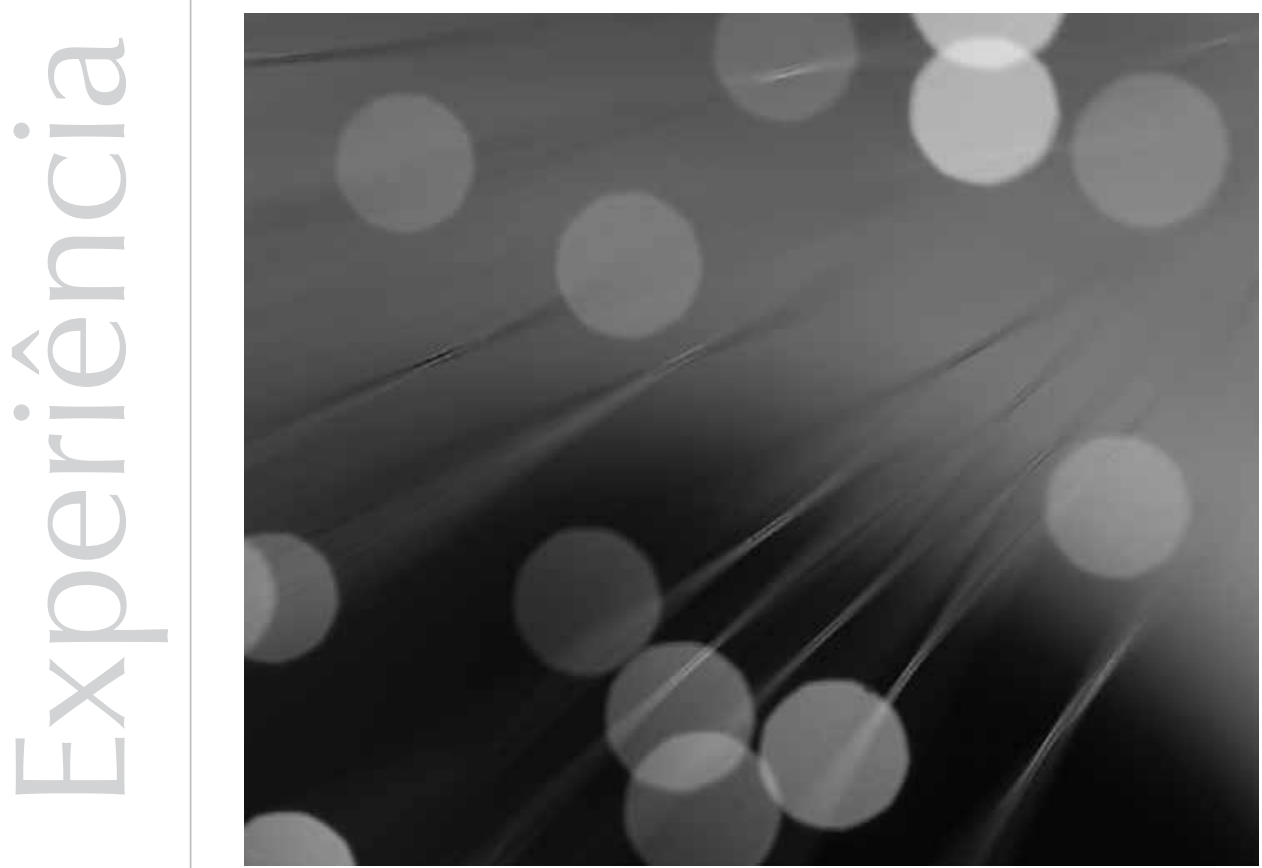
Resumo: Para reorientação da Atenção Básica, em 1998, o Ministério da Saúde (MS) adotou a Estratégia de Saúde da Família (ESF), que é operacionalizada por meio de equipes multiprofissionais em Unidades Básicas de Saúde (UBS), com o objetivo de substituir o modelo tradicional de atenção à saúde, tipicamente curativo e hospitalocêntrico. Este artigo analisa os desdobramentos da intervenção ocorrida durante o desenvolvimento do projeto de estágio Psicologia e Saúde Coletiva: Promovendo a Saúde na Comunidade, em uma UBS da cidade de Vitória (ES). Após um período de inserção na rotina e no contexto da unidade, elaboramos uma proposta de trabalho interdisciplinar dirigida ao grupo de pacientes composto por pessoas obesas, diabéticas e/ou hipertensas. Utilizamos mecanismos que possibilitaram trocas de experiências, como oficinas temáticas e passeios. No decorrer da intervenção, foi possível perceber a importância do trabalho interdisciplinar para que os objetivos propostos fossem alcançados. Verificamos muitas conquistas e algumas dificuldades, e constatamos que a Psicologia pode contribuir de forma efetiva para a promoção da saúde no contexto da ESF.

Palavras-chave: Psicologia. Sistema Único de Saúde. Interdisciplinaridade. Promoção da saúde.

Abstract: With the purpose of reorganizing health attention practices on a new basis and of replacing the traditional health model, whose focus was typically on healing and on hospital-centered practices, the Family Health Program (FHP) was created by the Brazilian Health Ministry in 1988. The FHP is operationalized by multiprofessional teams in Basic Health Units (BHU). This article aims to analyze the unfolding of an intervention developed in the course of a training program carried out under the project entitled "Psychology and collective health: promoting health in the community" conducted at a BHU in Vitória, ES. After inserting ourselves into the BHU routine and context for a certain time, we developed a proposal for an interdisciplinary program designed for a group of obese, diabetic and hypertensive patients. Mechanisms which promote the exchange of experiences such as thematic workshops and tours were created. During the intervention, it was possible to observe the importance of interdisciplinary work to reach the proposed objectives. We had improvements and certain difficulties, and concluded that Psychology can effectively contribute for the promotion of health in the context of FHP.

Keywords: Psychology. Brazilian National Health System. Interdisciplinarity. Health promotion.

Resumen: Para reorientación de la Atención Básica, en 1998, el Ministerio de la Salud (MS) adoptó la Estrategia de la Salud de la Familia (ESF), que es operacionalizada por medio de equipos multiprofesionales en Unidades Básicas de Salud (UBS), con el objetivo de sustituir el modelo tradicional de atención a la salud, típicamente curativo y hospitalocentrista. Este artículo analiza los despliegues de la intervención ocurrida durante el desarrollo del proyecto de práctica Psicología y Salud Colectiva: Promocionando la Salud en la Comunidad, en una UBS de la ciudad de Vitória (ES). Después de un período de inserción en la rutina y en el contexto de la unidad, elaboramos una propuesta de trabajo interdisciplinaria dirigida al grupo de pacientes compuesto por personas obesas, diabéticas y/o hipertensas. Utilizamos mecanismos que posibilitaron intercambios de experiencias, como talleres temáticos y paseos. En el transcurso de la intervención, fue posible notar la importancia del trabajo interdisciplinario para que los objetivos propuestos fuesen alcanzados. Verificamos muchas conquistas y algunas dificultades, y constatamos que la Psicología puede contribuir de forma efectiva para la promoción de la salud en el contexto de la ESF.

Palabras clave: Psicología. Sistema Único de Salud. Interdisciplinaridad. Promoción de la salud.

O curso de graduação em Psicologia da Universidade Federal do Espírito Santo (UFES) oferece estágios supervisionados em diversas áreas de atuação do psicólogo, entre eles o projeto Psicologia e Saúde Coletiva: Promovendo a Saúde na Comunidade (Dalbello-Araujo, 2000). O estágio tem duração de 225 horas distribuídas entre o nono e o décimo período de formação, precedido de uma disciplina obrigatória com carga horária de 60 horas que aborda os aspectos históricos e políticos do sistema de saúde brasileiro e discute as principais virtualidades e deficiências do Sistema Único de Saúde (SUS). O projeto se desenvolve desde 2000, e, por meio dele, mais de 80 alunos participaram do cotidiano de trabalho das Unidades Básicas de Saúde (UBS) da Rede Municipal de Atenção Básica. O projeto objetiva instrumentalizar os alunos do curso de Psicologia para a atuação no campo da saúde pública, contribuir para a construção do conhecimento em Psicologia no sentido de uma ressignificação dos conceitos de saúde física e mental, construir junto à comunidade atendida um espaço que 
possibilite a promoção da saúde e incentivar os profissionais da saúde para atuarem em abordagens coletivas.

O presente artigo visa a descrever e a analisar a experiência de intervenção psicológica grupal que ocorreu no referido estágio, durante o ano 2010, em uma das Unidades de Saúde da Família circunscritas à região de Maruípe, da cidade de Vitória, Espírito Santo.

\section{A Estratégia de Saúde da Família}

A Atenção Básica à Saúde (ABS) consiste na principal porta de entrada do SUS, e orienta-se pelos princípios da universalidade, da acessibilidade e da coordenação do cuidado, do vínculo e da continuidade, da integralidade, da responsabilização, da humanização, da equidade e da participação social (Brasil, 2006a). De acordo com a Política Nacional de Atenção Básica (Brasil, 2006b), a ABS caracteriza-se por um conjunto de ações no âmbito individual e coletivo, que abrangem a proteção, a recuperação e a promoção da saúde. Essas ações devem ampliar o seu escopo, visando os espaços além dos limites físicos das UBS.

Para reorientação dessa forma de atenção à saúde, o Governo Federal adotou a Estratégia de Saúde da Família (ESF), cujo enfoque das práticas está na família, e não no indivíduo. Tal estratégia é operacionalizada por meio de equipes multiprofissionais, habilitadas para desenvolver as atividades de promoção, proteção e recuperação (Brasil, 1997). Um dos objetivos dessa reorganização é substituir o modelo tradicional de atenção (Brasil, 1997, 2006a), tipicamente curativo e hospitalocêntrico. Para tanto, o território deve ser dividido possibilitando a sua cobertura pelas equipes, que contam com um médico da família ou generalista, enfermeiro, auxiliar de enfermagem e agentes comunitários de saúde (ACS). Além desses, outros profissionais podem ser incorporados às equipes (Brasil, 1997), como, por exemplo, psicólogos, assistentes sociais, farmacêuticos e educadores físicos. As equipes devem acompanhar um número definido de famílias (no máximo 4500 pessoas ou 1000 famílias), em um território determinado, o que facilita a aproximação entre usuários e profissionais e favorece a abordagem dos indivíduos de forma integral, pois a união de práticas e de saberes de profissionais de áreas distintas proporciona maior impacto sobre os diferentes fatores que interferem no processo de adoecimento da população (Araújo \& Rocha, 2007).

Dessa forma, as equipes são multiprofissionais e estão próximas à comunidade. No entanto, conforme afirmam Araújo e Rocha, essas características favorecem, mas não garantem um atendimento integral dos indivíduos. A modificação do modelo assistencial médicocentrado depende da mudança na forma de se produzir o cuidado e, também, na maneira como os profissionais agem entre si e com os usuários (Araújo \& Rocha, 2007). Na mesma linha, Franco e Merhy (2003) destacam que mesmo o trabalho direcionado a práticas multiprofissionais não garante que haverá a mudança do modelo de saúde hegemônico atualmente. Os autores discutem, ainda, que o Programa de Saúde da Família propõe uma mudança focada na estrutura e não opera de modo a alcançar os microprocessos do trabalho em saúde, por conseguinte, não transforma o cotidiano dos profissionais, que, em última instância, é o que define o perfil da assistência.

Como afirmam Franco e Merhy (2003), a gestão da ESF funciona a partir de um alto grau de normas regulamentadas verticalmente pelo Ministério da Saúde, sendo que, aos Municípios, cabe apenas seguir essas normas. Caso não façam o que está prescrito, deixam de receber financiamento, sem o qual fica inviável manter os serviços de saúde. Esse posicionamento pode acarretar 
"uma troca

profunda entre disciplinas, em que instrumentos, métodos e esquemas conceituais podem vir a ser integrados" (Artmann, 2001). um engessamento à ESF, o que seria mais condizente com o tradicional centralismo das políticas públicas do que com uma estratégia que se propõe à reorganização do modelo assistencial.

Diante disso, ressaltamos que o trabalho em equipe pode favorecer a comunicação e o intercâmbio de vivências e de informações entre os integrantes das equipes. No entanto, para que isso seja concretizado, é necessário maior investimento na formação contínua dos profissionais que compõem as equipes, ou seja, é importante que se realize o acompanhamento constante da formação e da capacitação dos profissionais, conforme prevê a Portaria $n^{\circ}$ 648/2006, que rege a Política Nacional de Atenção Básica (Brasil, 2006a).

\section{Trabalho interdisciplinar em equipe de ESF}

Partindo do que foi exposto, consideramos que, para efetivação do trabalho em Saúde da Família, é necessário que se construa um projeto comum de assistência no qual os profissionais trabalhem de forma complementar. Dessa maneira, a comunicação e a interação entre os profissionais, e entre estes e a comunidade, são importantes ferramentas para a atenção integral da clientela (Araújo \& Rocha, 2007). Entretanto, o trabalho das equipes de Saúde da Família é frequentemente permeado pela fragmentação do conhecimento humano, e, para superar essa situação, seria necessária a busca por uma atuação baseada na visão globalizada do processo saúde-doença. Para dar conta da complexidade desse processo, é preciso trabalhar de forma interdisciplinar, que tem como característica "uma troca profunda entre disciplinas, em que instrumentos, métodos e esquemas conceituais podem vir a ser integrados" (Artmann, 2001).
A interdisciplinaridade difere da multidisciplinaridade e da transdiciplinaridade, uma vez que se caracteriza por uma integração entre as disciplinas. Na multidisciplinaridade, um mesmo objeto é investigado por diversas disciplinas, sem que isso implique a construção de métodos e de conceitos comuns a elas (Santos, 2007). A transdisciplinaridade, por outro lado, transpõe os limites das disciplinas. De acordo com Domingues (2003), nesta há uma construção de conhecimento com o mais profundo entrelaçamentos das disciplinas envolvidas, fazendo com que sejam derrubadas as demarcações que as separam. O autor afirma, ainda, que esta última é uma utopia, mas que deve ser perseguida.

Diante disso, a interdisciplinaridade não deve ser entendida como uma panaceia, mas como uma possibilidade de contribuição para maior resolutividade dos problemas enfrentados, assim como para a identificação de um objeto comum a vários profissionais. Embora o trabalho interdisciplinar seja difícil de ser concretizado, ele tem grande importância, pois nenhuma disciplina isoladamente explica a totalidade do ser humano e suas relações sociais (Santos, 2000).

Segundo Campos e Belisário (2001), entre os diferentes problemas que surgem com a implantação do PSF, o mais grave é a carência de profissionais, em termos quantitativos e qualitativos, para atender a essa nova necessidade. Em outras palavras, há uma dificuldade em se efetivar o trabalho interdisciplinar em função da formação dos profissionais, que ainda é atravessada pela cultura de especialismos.

\section{A atuação do psicólogo na ESF}

Conforme mencionado anteriormente, além dos profissionais que compõem a equipe mínima da ESF, outros profissionais podem ser incorporados às unidades, dentre eles, o psicólogo. Com relação à atuação desse 
profissional junto às equipes multidisciplinares, estudos (Bittencourt \& Mateus, 2006; Soares, 2005) têm demonstrado que profissionais das equipes avaliam que o papel do psicólogo no contexto de ESF se faz muito importante. Esses trabalhos demonstram que a atuação desse profissional pode estar voltada tanto para atividades com grupos específicos, visitas domiciliares e orientação das equipes quanto para questões técnicas, atendimento individual, avaliação da demanda, estudos de caso e incentivo para facilitar a comunicação entre a comunidade e a equipe de saúde.

O trabalho do psicólogo nos serviços de Atenção Primária à Saúde também deve estar voltado para atividades de promoção da saúde, visando à melhoria da qualidade de vida da população. Santos, Quintanilha e Dalbello-Araujo (2010) demonstraram que as concepções dos profissionais psicólogos de uma região da cidade de Vitória/ES a respeito da promoção da saúde estão ligadas à educação em saúde, à prevenção de doenças por meio de mudança de hábitos e de comportamentos individuais e à promoção de qualidade de vida a partir de grupos e de trocas de ideias. As autoras afirmam, ainda, que a atuação dos psicólogos revelou uma busca por afirmação de novas práticas no campo da saúde, entre elas, aquelas ligadas às propostas do SUS e, em consequência, às que envolvem a promoção da saúde.

As referidas concepções muitas vezes são decorrentes do processo de formação durante a graduação. De acordo com Soares, é importante que os órgãos formadores repensem o profissional que estão formando. Há urgência em se repensar, também, os currículos em vigor nos cursos de Psicologia, incluindo a conscientização em relação à realidade, e a mudança dos paradigmas que os têm norteado. A autora ressalta que, a partir das possibilidades de atuação do psicólogo na ESF, "seja qual for a perspectiva teórico-metodológica adotada que sustente a prática, para se alcançar os resultados positivos esperados, há que se ter disposição, vivacidade e adotar sem medo um novo olhar" (Soares, 2005, p. 598).

Apesar do reconhecimento da importância da participação do psicólogo no contexto de ESF, Soares chama a atenção para as dificuldades existentes para a inserção do psicólogo nas equipes multidisciplinares. A autora afirma que há falta de clareza em relação à função ou mesmo à importância do trabalho da Psicologia nesse contexto. Assim, é necessário que haja esclarecimento do papel do psicólogo tanto para a população quanto para as equipes. Uma das formas de propiciar o conhecimento daquilo que a Psicologia pode oferecer para ampliar a promoção da saúde é, sem dúvida, atuar de forma conjunta com outros profissionais. Nessas atividades, quase sempre coletivas, ao mesmo tempo em que se produz o cuidado ou mesmo a educação em saúde, estabelecem-se formas de intervenção conjunta em que cada profissional mostra sua forma peculiar de agir. Assim, tendo a atividade como mediadora, os diferentes modos de pensar e as diferentes concepções sobre o processo saúde-doença vão sendo tecidos, o que pode contribuir para um trabalho interdisciplinar (França \& Viana, 2006).

\section{Relato de experiência no estágio Psicologia e Saúde Coletiva: Promovendo a Saúde na Comunidade}

A USF na qual foi desenvolvido o estágio é a segunda maior do Município de Vitória, abrange uma população de 15.086 pessoas (Bastos, Fraga, Fejoli, Zavaris, \& Azoury, 2010) e conta com o trabalho de seis equipes divididas em trinta microáreas, das quais oito são consideradas de alto risco devido à grande vulnerabilidade social. No período em que foi realizada a intervenção, seis microáreas não 
contavam com a presença de ACS, o que prejudicava a atuação das equipes como um todo.

As atividades relativas ao estágio se estenderam por nove meses, entre março e dezembro de 2010. No decorrer do primeiro semestre, foram desenvolvidas atividades de acompanhamento do cotidiano do serviço, tais como reuniões das equipes, grupo de adolescentes, grupo composto por obesos, diabéticos e/ou hipertensos, ação comunitária, visita domiciliar, reunião no Centro de Referência de Assistência Social (CRAS) e atendimento psicológico.

Nesse sentido, o primeiro momento de inserção na USF permitiu a vivência da unidade em questão e a observação das nuances existentes entre o SUS que se conhece por meio dos documentos e aquele que se experimenta no cotidiano. Em muitos momentos, foi possível notar o conflito que se apresentava quando o prescrito não atendia às reais necessidades demandadas. Além disso, o contato com a comunidade possibilitou conhecer os seus contextos social, cultural e econômico e elaborar uma intervenção condizente com este.

Esse período foi importante para que fosse elaborada a proposta de intervenção, que visava a responder à necessidade apresentada pelo serviço, isto é, a participação da Psicologia em atividades coletivas. Essa participação teve como objetivo promover uma intervenção no grupo que não enfocasse exclusivamente as questões biológicas, mas que trouxesse uma compreensão mais ampla do processo saúde-doença.

A inserção para o desenvolvimento do trabalho propriamente dito se deu em um grupo do qual participaram pessoas com sobrepeso, obesas, diabéticas e/ou hipertensas. Naquele momento, estavam à frente do grupo uma educadora física, uma médica e uma auxiliar de enfermagem. Tal grupo já era realizado há aproximadamente dois anos, período no qual eram desenvolvidas atividades como palestras, acompanhamento do peso dos participantes, aferição da pressão arterial e do índice glicêmico. Os encontros aconteciam semanalmente, no auditório da Unidade de Saúde, e a participação contínua dos usuários não era exigida. Assim, o número de cadastrados no grupo era significativamente maior do que a presença neste. Dentre os participantes, 92,9\% eram mulheres, e a idade variava entre 34 e 76 anos.

A demanda da intervenção psicológica no grupo configurou-se no sentido da importância em acrescentar conhecimentos capazes de trabalhar os aspectos subjetivos emergentes naquele contexto. Com essa finalidade, nossas práticas se direcionaram para contribuir com as ações educativas já desenvolvidas e, principalmente, para promover espaços de fala e de acolhimento daquilo que os participantes traziam como problemática. Além disso, o trabalho com os profissionais de áreas diferentes apresentouse como uma forma de tornar mais claras as possibilidades de atuação de um psicólogo dentro da UBS por meio do trabalho interdisciplinar. A seguir, vamos relatar como se deu a intervenção no referido grupo. Assim, nossa intervenção foi realizada em um grupo programático tradicional coordenado por profissionais de duas áreas diferentes: Educação Física e Medicina. Buscando um trabalho interdisciplinar, priorizamos o diálogo entre essas disciplinas, para que assim pudéssemos construir um método de intervenção que alcançasse um objetivo comum: trazer mais qualidade de vida para a população atendida. Para isso, optamos por utilizar oficinas temáticas como instrumento de intervenção. Cada oficina deveria ser elaborada conjuntamente pelos profissionais envolvidos no grupo, porém encontramos alguns percalços quanto à comunicação 
dentro da equipe. A seguir, descreveremos detalhadamente como se deu a intervenção bem como as conquistas e os desafios que encontramos nesse percurso.

\section{Intervenção}

Observamos o funcionamento do grupo durante os meses de junho e julho e realizamos a intervenção nos meses de agosto, setembro e outubro, totalizando 11 encontros. Durante a intervenção, utilizamos mecanismos que possibilitaram trocas de experiências, como oficinas temáticas e passeios. As oficinas foram realizadas semanalmente, com duração de aproximadamente uma hora, no auditório da USF. Realizamos, ainda, um café da manhã e uma caminhada da unidade até um parque municipal de Vitória. Tais atividades foram desenvolvidas com a participação de outros profissionais que estavam à frente do grupo. Ao longo do trabalho, foi preciso reavaliar a proposta a fim de adequá-la ao que surgiu no processo.

Na oficina Fotografia, realizamos atividades como maquiar e tirar fotos dos participantes, e finalizamos com uma discussão sobre essa experiência. Buscamos, assim, favorecer um espaço de reflexão acerca da imagem corporal que os participantes tinham deles mesmos. Para o encontro seguinte, selecionamos algumas fotos, e, por meio delas, trabalhamos a oficina Porta-retrato, na qual os participantes confeccionaram os seus porta-retratos.

A partir do que o grupo trouxe como problemática, elaboramos a oficina $\mathrm{Em}$ busca de equilíbrio nas relações e na vida, em que utilizamos como dispositivo figuras em forma de coração e em forma de corpo humano, com o objetivo de propiciar uma conscientização de como estava o equilíbrio das diversas áreas do espaço vital do participante e também acerca das atitudes que poderiam ser tomadas na busca por mudanças possíveis e necessárias. Em um primeiro momento, foi realizado um exercício corporal que propiciasse o relaxamento dos participantes. Em seguida, solicitamos que refletissem a respeito das emoções e do corpo e, depois, que atribuíssem notas (de zero a dez) a essas duas áreas.

Durante a nossa intervenção, as características específicas do grupo, que é composto basicamente por pessoas com restrições alimentares, foram levadas em consideração. Dessa maneira, a oficina Dieta pela vida foi conduzida no sentido de estimular a compreensão dos participantes do ato de alimentar-se, além de promover uma discussão sobre o impacto da dieta na vida de cada um deles. Para isso, usamos materiais como figuras de alimentos e figuras de bebidas. Solicitamos aos participantes que montassem um prato com os alimentos que desejassem e, em seguida, que montassem um prato com aquilo que acreditassem ser o correto. Ao final da atividade, refletimos a respeito dos sentimentos que apareceram durante a preparação dos pratos.

Na oficina Como me vejo, solicitamos aos participantes que se olhassem em um espelho e observassem a sua própria imagem. Nosso objetivo era o de oportunizar o relato dos sentimentos que emergiam a respeito das mudanças físicas que haviam surgido em decorrência do passar do tempo. Após essa oficina, averiguamos a necessidade de trabalhar o uso que os participantes faziam do tempo em seu cotidiano, e, assim, no encontro seguinte, realizamos a oficina Relógio do cotidiano. Disponibilizamos figuras de relógios, papel, lápis de cor, canetinha e giz de cera, e pedimos que desenhassem um relógio, marcando em suas horas as tarefas que realizavam no dia a dia. Sugerimos, então, que os participantes refletissem acerca de como e com quem o seu tempo era passado. 
A nossa opção por utilizar as oficinas como estratégia metodológica se justifica por acreditarmos que elas tenham potências terapêuticas e pedagógicas, pois são instrumentos que facilitam a elaboração de questões subjetivas, interpessoais e sociais, assim como eliciam um processo de aprendizagem que parte de reflexões sobre as vivências; facilitam, ainda, uma elaboração do conhecimento sobre o mundo e sobre si mesmo (Afonso, 2006).

É importante ressaltar que a oficina deve estar de acordo com a experiência dos participantes (Afonso, 2006). Dessa maneira, as oficinas que realizamos foram elaboradas a partir do que o grupo apresentou como demanda. Além disso, procuramos ficar atentas ao desenvolvimento que o grupo dava à atividade, assim, não ficamos presas no que estava previsto em detrimento do que foi levantado como questão.

\section{Conquistas e dificuldades}

Ainda que o convite para nossa atuação junto ao grupo tenha sido verbalizado apenas por um profissional, em várias outras ocasiões, os demais afirmaram a importância da participação da Psicologia em atividades coletivas. Tal conduta corrobora os estudos de Bittencourt e Mateus (2006) e de Soares (2005), que revelam a importância dada pelos profissionais da Atenção Básica à presença do psicólogo na ESF. A intervenção no grupo, portanto, foi entendida como uma possibilidade para atender à demanda que foi colocada, e, no decorrer da intervenção, foi possível verificar os benefícios proporcionados pelas atividades. Constatamos que, a partir dos conteúdos evocados nos encontros, o próprio grupo pôde problematizar aspectos de sua vida e sugerir soluções, e várias foram as oportunidades em que o grupo acolheu os sentimentos vivenciados pelos demais participantes, o que denota a construção do sentimento de grupalidade entre eles.
As atividades realizadas propiciaram uma reflexão acerca dos modos de vida. Dessa maneira, verificamos, por meio de relatos e de comportamentos dos participantes, que as atividades atingiram o seu objetivo no sentido em que oportunizaram um processo de reflexão sobre amplos aspectos para além das doenças que os trouxeram ali inicialmente. Assim, as oficinas facilitaram a elaboração de questões subjetivas, interpessoais e sociais dos participantes do grupo, e possibilitaram o conhecimento sobre si mesmo (Afonso, 2006). Como exemplo disso, verificamos que algumas pessoas passaram a cuidar melhor da aparência e a valorizar mais a sua presença no grupo. Uma das participantes declarou que não foram realizadas apenas palestras, mas também atividades que promoviam uma melhora na autoestima. Além disso, na oficina Como me vejo, constatamos que alguns usuários não conseguiam olhar-se no espelho, o que propiciou a emergência de conteúdos significativos para repensar sua condição no mundo. Em outra oficina, Relógio do cotidiano, os participantes refletiram sobre o uso que faziam do tempo, e concluíram que dedicavam muito tempo ao trabalho e à família em detrimento do cuidado com eles mesmos. Assim, as atividades realizadas na Unidade de Saúde extrapolaram o costumeiro foco na doença e puderam agregar outros aspectos da vida dos usuários, como preconizado pela Política Nacional de Promoção de Saúde (Brasil, 2006b).

Além das conquistas descritas, no decorrer dos encontros, também surgiram obstáculos. O maior deles diz respeito à dificuldade para efetivar um trabalho interdisciplinar; o estabelecimento de um horário em comum no qual os envolvidos na coordenação do grupo pudessem encontrar-se para planejar e avaliar as atividades se colocou como um dos entraves. Essa incompatibilidade de horário acarretou a falta de planejamento conjunto das atividades realizadas, assim como a ausência de um espaço para discutir os seus 
resultados. Esse impasse revela a rigidez da estrutura de trabalho da ESF, já apontada por Franco e Merhy (2003), que dificulta a comunicação entre os profissionais. Diante disso, podemos afirmar que, ainda que as atividades no grupo tenham transcorrido de maneira bastante satisfatória, não foi possível efetivarmos o trabalho interdisciplinar pretendido.

A dificuldade em concretizar o trabalho interdisciplinar tem em suas bases, ainda, na formação dos profissionais, pois, conforme afirma Santos, "o conhecimento está dividido sobre a forma de disciplinas" (2007, p. 52). A autora ressalta, também, que esse trabalho realizado por disciplinas diferentes pode acarretar a tentativa de uma disciplina se sobrepor às demais. Acreditamos que, em trabalhos realizados conjuntamente por profissionais de áreas diversas, como o que propúnhamos, essa sobreposição de saberes acarreta prejuízos, inclusive, inviabilizando o diálogo e a comunicação entre os profissionais. Compreendemos que essa dificuldade pode ser reflexo da formação dos profissionais, ainda muito marcada pela cultura de especialismos (Campos \& Belisário, 2001).

Outro entrave encontrado diz respeito ao desconhecimento acerca das possibilidades de contribuição da Psicologia para atividades coletivas, assim como de seus instrumentos e objetivos, o que, algumas vezes, acarretou o mau uso destes últimos.

A oficina Dieta pela vida, por exemplo, teve como objetivo promover uma ampla discussão sobre os reflexos da dieta na vida dos participantes. No entanto, o material que havia sido por nós confeccionado, em alguns momentos, foi utilizado por outros profissionais de forma estritamente educativa, ou seja, nosso instrumento não proporcionou o espaço que pretendíamos para que os participantes falassem sobre como se sentiam diante da necessidade de fazer uma dieta, em virtude de seus problemas de saúde. Ao contrário, devido à falta de comunicação e de planejamento conjunto, ele foi usado de forma repreensiva, uma vez que os participantes não se alimentavam da maneira considerada, pelo saber médico, correta.

Esses dados se assemelham aos encontrados no estudo de Soares (2005), que mostram incompreensão em relação à função ou mesmo à importância do trabalho da Psicologia no contexto da ESF. Entendemos que tais fatos também se devem às dificuldades de comunicação entre os profissionais atuantes no grupo, pois, de acordo com o que afirmam Araújo e Rocha (2007), a comunicação e a interação entre os profissionais são importantes para a prática de atenção integral à saúde da população. Além disso, segundo Cardoso (2002), os psicólogos devem atuar junto aos outros profissionais da equipe de forma a integrar esforços, a estimular a reflexão e a troca de informações sobre a população atendida.

Constatamos, também, que a falta de comunicação que acarretou prejuízos ao trabalho se deve, em parte, ao modo como a ESF é estruturada. A alta centralização e a verticalização da gestão dessa estratégia, como apontam Franco e Merhy (2003), impedem a existência de espaços para que os profissionais saiam do que está previsto. Dito de outra forma, ainda há um aprisionamento ao que está prescrito, impedindo os profissionais de inovarem e de adequarem a sua prática à realidade da vida da comunidade onde trabalham.

\section{Considerações finais}

A experiência que acabamos de relatar mostra a importância de um trabalho interdisciplinar no contexto da ESF, pois este favorece a abordagem de forma integral da clientela 
atendida. No entanto, expõe, também, algumas dificuldades para a implementação dessa forma de trabalho. Nesse sentido, ressalta a importância de maior comunicação entre os profissionais assim como a necessidade de uma formação contínua destes.

As atividades realizadas durante a intervenção não enfocaram a doença, mas, ao contrário, enfatizaram as potencialidades daqueles que frequentavam o grupo. $\mathrm{O}$ uso de oficinas como metodologia se mostrou eficaz, uma vez que estabelece como princípio o fornecimento, aos participantes, de um espaço para elaboração de modos de agir, de pensar e de sentir por meio da comunicação, da conversa.

Porém, de acordo com nossa avaliação, o trabalho poderia ter se dado de forma ainda mais satisfatória, caso tivesse ocorrido melhor planejamento e avaliação das atividades em conjunto com os outros profissionais envolvidos com o grupo. Nesse sentido, é fundamental que a gestão da ESF esteja configurada de modo a incentivar as atividades interdisciplinares e a permitir que os profissionais tenham maior autonomia em relação à gestão do próprio trabalho.

Diante do que foi exposto, é possível observar que o atendimento da clientela por meio de grupos interdisciplinares é uma iniciativa válida, pois, mesmo com as dificuldades de comunicação enfrentadas, foi prestado um atendimento à população que não se restringiu ao saber de uma única disciplina. A oficina Fotografia foi um bom exemplo de efetivação do trabalho interdisciplinar. Houve uma integração entre os membros da equipe, e, principalmente, o alcance do objetivo comum de maior qualidade de vida para os participantes.

Ao analisar a experiência descrita, concluímos que o trabalho de psicólogos junto a atividades coletivas tem importância e é pertinente às ações de promoção da saúde no contexto de USF. Assim, por meio deste artigo, esperamos contribuir para as práticas de promoção da saúde e, ainda, possibilitar uma reflexão sobre formas de gestão e do trabalho interdisciplinar. Tal problemática é dotada de uma complexidade que não se esgota na experiência apresentada. Devido a isso, julgamos necessária a realização de estudos que relacionem as formas de gestão da ESF com a realização dos trabalhos interdisciplinares e com as ações de promoção da saúde. 


\section{Leandra Lúcia Moraes Couto}

Mestranda em Psicologia pela Universidade Federal do Espírito Santo, Vitória - ES - Brasil.

E-mail: leandrabj@hotmail.com

\section{Polyana Barbosa Schimith}

Mestre em Psicologia pela Universidade Federal do Espírito Santo, Vitória - ES - Brasil.

E-mail: ninha.bs@gmail.com".

\section{Maristela Dalbello-Araujo}

Doutora em Psicologia pela Universidade Federal do Espírito Santo e docente da Universidade Federal do Espírito Santo, Vitória - ES - Brasil.

E-mail: dalbello.araujo@gmail.com

\section{Endereço para envio de correspondência:}

Universidade Federal do Espírito Santo, Centro de Ciências Humanas e Naturais, Departamento de Psicologia.

Av. Fernando Ferrari, s/n, Goiabeiras. CEP: 29060-900. Vitória, ES.

Recebido: 11/01/2012, 1a Reformulação: 13/01/2013 Aprovado: 31/01/2013. 
Afonso, M. L. M. (2006). Oficinas em dinâmica de grupo: um método de intervenção psicossocial. In M. L. M. Afonso (Org.). Oficinas em dinâmica de grupo: um método de intervenção psicossocial (pp. 9-61). São Paulo: Casa do Psicólogo.

Araújo, M. B. S., \& Rocha, P. M. (2007). Trabalho em equipe: um desafio para consolidação da estratégia de Saúde da Família. Ciência e Saúde Coletiva, 12(2), 455-464.

Artmann, E. (2001). Interdisciplinaridade no enfoque intersubjetivo habermasiano: reflexões sobre planejamento e AIDS. Ciência \& Saúde Coletiva, 6(1), 183-195.

Bastos, E. M., Fraga, I. S., Fejoli, M. M., Zavaris, N. A., \& Azoury, T. C. C. (2010). Diagnóstico situacional do território da unidade de saúde de Consolação. Trabalho apresentado ao Programa de Pós-Graduação em Saúde Coletiva do Centro de Ciências da Saúde, Universidade Federal do Espírito Santo, Vitória, ES.

Bittencourt, R. A. A., \& Mateus, M. L. F. (2006). Possibilidades de atuação do psicólogo no programa Saúde da Família: a experiência de Bonito-MS. Psicologia: Ciência e Profissão, 26(2), 328-343.

Brasil. Ministério da Saúde. (1997). Saúde da Família: uma estratégia para reorientação do modelo assistencial. Brasília, DF: Ministério da Saúde. Recuperado em jul. 2012, de http:// bvsms.saude.gov.br/bvs/publicacoes/cd09_16.pdf

Brasil. Ministério da Saúde. Secretaria de Atenção à Saúde. (2006a). Departamento de Atenção Básica. Política Nacional de Atenção Básica. Brasília, DF: Ministério da Saúde.

Brasil. Ministério da Saúde. (2006b). Secretaria de Vigilância em Saúde. Política Nacional de Promoção da Saúde. Brasília, DF: Ministério da Saúde.

Campos, F. E., \& Belisário, S. A. (2001). O Programa de Saúde da Família e os desafios para a formação profissional e a educação continuada. Interface - Comunicação, Saúde, Educação, 5(9), 133-141.
Cardoso, C. L. (2002). Inserção do psicólogo no Programa Saúde da Família. Psicologia: Ciência e Profissão, 22(1), 2-9.

Dalbello-Araujo, M. (2000). Psicologia e saúde coletiva: promovendo a saúde na comunidade. Projeto de estágio supervisionado. Departamento de Psicologia, Universidade Federal do Espírito Santo, Vitória, ES.

Domingues, I. (2003). Humanidade inquieta. Revista da Universidade Federal de Minas Gerais, 1(2), (Entrevista). Recuperado em 27 dez., 2012, de https://www.ufmg.br/ diversa/2/entrevista.htm

Franco, T. B., \& Merhy, E. E. (2003). Programa de Saúde da Família (PSF): contradições de um programa destinado à mudança do modelo tecnoassistencial. In E. E. Merhy, J. H. M. Magalhães, J. Rimoli, T. B. Franco \& W. S. Bueno, O trabalho em saúde: olhando e experienciando o SUS no cotidiano ( 3a ed., pp. 53-124). São Paulo: Hucitec.

França, A. C. P., \& Viana, B. A. (2006). Interface psicologia e Programa Saúde da Família - PSF: reflexões teóricas. Psicologia: Ciência e Profissão, 26(2), 246-257.

Santos, S. S. C. (2000). Enfermagem gerontológica: reflexão sobre o processo de trabalho. Revista Gaúcha de Enfermagem, $21(2), 70-86$.

Santos, M. S. (2007). Integração e diferença em encontros disciplinares. Revista Brasileira de Serviço Social, 22(65), 51-60.

Santos, K. L., Quintanilha, B. C., \& Dalbello-Araujo, M. (2010). A atuação do psicólogo na promoção da saúde. Psicologia: Teoria e Prática, 12(1), 181-196.

Soares, T. C. (2005). "A vida é mais forte do que as teorias" - o psicólogo nos Serviços de Atenção Primária à Saúde. Psicologia: Ciência e Profissão, 25(4), 590-601. 